\title{
EL MUNDO DEL TRABAJO Y LA PRECARIEDAD LABORAL DE LOS COMUNICADORES Y PERIODISTAS
}

\begin{abstract}
Evelyn Daniela Caminos ${ }^{1}$
Sumario: I.-Introducción. II.-Comunicadores sociales y periodistas: estudios que demuestran su realidad laboral actual. III.-Abordaje de la problemática desde el enfoque del derecho del trabajo. IV.Definición por la OIT del trabajo precario. V.-Diferentes formas de condiciones y contrataciones laborales precarias que se vienen propagando. VI.-Marco legal que contempla a los comunicadores sociales y periodistas. VII.-Conclusión.
\end{abstract}

\begin{abstract}
Resumen: El mundo del trabajo en la actualidad está atravesando acelerados cambios y una revolución tecnológica, denominada Cuarta Revolución Industrial; a la par se vivencia una desregulación de los mercados laborales y el aumento de la flexibilidad. Las nuevas formas de organización del trabajo afectan a los trabajadores en general y a los comunicadores y periodistas en particular, que como un subconjunto de trabajadores profesionales mantienen pluriempleo, bajos salarios, y condiciones laborales de precarización y flexibilización. El objetivo del artículo es conocer el mercado laboral de estos profesionales, el marco legal que los protege y la desregulación de sus nuevas formas de trabajo. En tal sentido, en primer lugar, se analiza el corpus bibliográfico, y luego desde la óptica del derecho del trabajo y la seguridad social, las nuevas modalidades de contratación y relaciones de empleo. Por último, se describe la normativa existente en la materia que se caracteriza por sus vacíos legislativos y por la falta de regulación legal.
\end{abstract}

Palabras clave: Precariedad. Comunicadores. Periodistas. Condiciones. Modalidades.

\section{I.- Introducción}

El mundo del trabajo en la actualidad está atravesando acelerados cambios y una revolución tecnológica, denominada Cuarta Revolución Industrial; a la par se vivencia una desregulación de los mercados laborales y el aumento de la flexibilidad, lo que trae aparejada una profunda crisis producto de la elevada informalidad y precariedad laboral.

La Organización Internacional del Trabajo (OIT), en el documento de final del Simposio de los trabajadores sobre políticas y reglamentación para luchar contra el empleo preca-

1 Abogada (UNC); empleada en el fuero laboral del Poder Judicial de la Provincia de Córdoba; maestranda en Derecho del Trabajo y Relaciones Laborales Internacionales (UNTREF); doctoranda en Administración y Política Pública (IIFAP - UNC),adscripta en la Catedra de Derecho del Trabajo (UCC); miembro Investigadora SECyT de la UNC. E-mail de contacto: caminosevelyn@gmail.com 
rio, concluye que: "Un inimaginable número de trabajadores en todo el mundo padece condiciones laborales precarias, inseguras, inciertas e impredecibles. Las cifras de desempleo provocan preocupación por sí mismas, pero ni siquiera logran abarcar a una mayoría más amplia de personas que trabajan pero que no tienen un empleo decente con salarios dignos, futuro estable, protección social y acceso a derechos fundamentales" $(2012,3)$.

Los periodistas y comunicadores sociales, como trabajadores, no están exentos del fenómeno mundial de cambios en el mundo del trabajo, que obedecen a cuestiones estructurales y coyunturales que afectan simultáneamente a las condiciones de trabajo y el mercado laboral.

Entre las causas estructurales, podemos mencionar el advenimiento del nuevo capitalismo financiero o posindustrial, con preeminencia del trabajo inmaterial y la economía de los servicios (Castel, 2012, 16). La crisis de los dos sistemas tradicionales de producción (el taylorismo y el fordismo) así como el auge actual del toyotismo como modalidad de desarrollo de los servicios. La irrupción de las new economy o sociedad de la información, en el que las nuevas tecnologías de la Comunicación y de la Información (NTIC), adquieren un rol dominante (Rodríguez y Sánchez, 2004, 13).

Entre las causas coyunturales, podemos mencionar cuestiones como las políticas neoliberales laborales, económicas, financieras y sociales implementadas por los países que respondieron al Consenso de Washington en la década de los noventa (Castel, 2012; Velásquez Pinto, 2016 en Novick, 2018), así como también la saturación actual del mercado del comunicador. Como fenómeno de los últimos meses, se destaca la irrupción de la pandemia COVID-19, que ha revolucionado el modo en que nos relacionamos y la forma de trabajar. Las restricciones a la movilidad de personas y factores productivos han obligado a muchos sectores y organizaciones a implementar con urgencia procesos de digitalización para hacer frente el nuevo panorama y repensar el futuro del trabajo, entre ellos el sector de los comunicadores y periodistas.

\section{Comunicadores sociales y periodistas estudios que demuestran su realidad laboral actual}

Como un subconjunto de trabajadores profesionales, los comunicadores y periodistas mantienen pluriempleo, bajos salarios, y condiciones laborales de precarización y flexibilización. Los estudios presentan a un comunicador que trabaja más de 8 horas diarias, con descansos reducidos, horarios irregulares, trabajos nocturnos y bajos salarios. Con síntomas de ansiedad causada por la necesidad de estar a la vanguardia de la profesión, con polivalencia de tareas, multifuncionalidad, ejerciendo una profesión insegura, y con tendencia al autoempleo, al subcontrato o al trabajo informal, con un perfil policompetente y multifunción (Blanco, 2016; Baranchuk, 2016; Chinea Rodríguez, 2013; Lagos y Cabalin, 2009; Martínez, 2019; Ufarte Ruiz, 2011).

Haciendo referencia al derecho colectivo, algunos de los estudios analizados dan cuenta de la falta de sindicalización o asociación de los profesionales, y desregulación legal general y de convenios sectoriales (Ufarte Ruiz, 2011). Las causas de la falta de sindicalización y colegiación pueden deberse a innumerables factores, entre ellos, a que los comunicadores se autoperciben desde la lógica de las profesiones liberales, y que existen diferencias hacia su interior a causa del gran abanico de ámbitos de desempeños donde pueden desarrollarse. 
Otra causa de la no sindicalización o asociación puede ser que su actividad, sobre todo la de aquellos que se emplean en los medios de comunicación, está íntimamente relacionada con los derechos constitucionales de libertad de expresión y libertad de pensamiento, lo que históricamente ha ocasionado que se intervengan y persigan ideológicamente a las organizaciones que los agrupan. Por último, otra explicación posible sería la falta de respuesta de las organizaciones a los cambios tecnológicos que han modificado en un corto tiempo las formas de producción (Baranchuk,2016; Martínez, 2019).

Veamos las conclusiones de algunos estudios recabados. Del Informe Anual de la profesión periodística, realizado en España en el 2017, surge que casi la mitad (45,5\%) de los españoles profesionales del área encuestados (comunicadores y periodistas), consideran que el principal problema que tienen ante sí es el desempleo y la precariedad labo$\mathrm{ral}^{2}$; y que esta última se manifiesta en altas tasas de contrataciones temporales, bajos salarios y las desmesuradas participaciones de colaboradores o pasantes, además de la desregulación legal.

Por otro lado, se encuentra el estudio de Ufarte Ruiz (2008) -de carácter exploratorio, descriptivo, de muestra simple no probabilística ${ }^{3}$, que intenta dar cuenta de la precariedad de los periodistas almarienses en la prensa escrita. La investigadora señala que en los medios impresos es donde la precariedad y las presiones se desarrollan con mayor intensidad.

En el desarrollo de su tesis, indica que la precariedad también se debe a que no existe regulación laboral sectorial, ni estatal, ni andaluza, ni provincial en la radio y televisión de la comunidad autónoma. Por otro lado, nombra las tendencias desreguladoras en el ámbito laboral, la baja cobertura de los convenios sectoriales estatales, la falta de organización sindical, y del nuevo fenómeno de externalización de la actividad a través de la subcontratación y el uso desmedido de becarios y pasantes de prácticas profesionales.

Los resultados del estudio de Ufarte Ruiz arrojan que casi el $80 \%$ piensa que el periodismo es una profesión precaria. "En cuanto a las razones que argumentan, los bajos salarios y las excesivas jornadas de trabajo encabezan la lista con un $29 \%$ y un $21 \%$ respectivamente. Seguidamente, destacan como principales causas la inestabilidad en el puesto de trabajo y los excesivos contratos temporales (11\%), la falta de respeto a los derechos laborales de los trabajadores (9\%), el intrusismo laboral (6\%) y por último el abuso del trabajo realizado por los estudiantes en prácticas (3\%)" (Ufarte Ruiz 2011, 457-458).

Otro estudio sobre el tema es el que ha llevado adelante Chinea Rodríguez (2013), quien realizó un estudio longitudinal en el que analiza las trayectorias biográficas de 10 directores de plataformas vinculadas al sector de la comunicación, en Islas Canarias, España.

\footnotetext{
2 Fuente: Informe Anual de la Profesión Periodística, 2017. APM: Encuesta profesional, 2017. Cifras en porcentajes. Base: 1756. Este informe utiliza una pluralidad de fuentes. En primer lugar, por su importancia para reflejar la situación del periodismo en España, la Encuesta Profesional de la Asociación de la Prensa de Madrid (APM), que, a través de una empresa especializada, se envía a más de 13500 periodistas. La Federación de Asociaciones de Periodistas de España (FAPE) y con la colaboración de la Federación de Sindicatos de Periodistas (FeSP), el Sindicato de Periodistes de Catalunya (SPC), y las secciones de periodistas de la Unión General de Trabajadores (UGT), y la Confederación Nacional del Trabajo (CNT), que, mediante un sistema de enlace abierto, hicieron llegar la encuesta a sus afiliados También se ha enviado esta encuesta a un grupo de periodistas participantes en el programa Primer Empleo de la APM. En total se recibieron 1756 encuestas cumplimentadas, lo que supone un porcentaje de respuesta superior al $13 \%$ y una base de contestaciones suficiente para conocer en profundidad la situación del colectivo de periodistas de España.

3 De los 99 periodistas a los que se les mandó el cuestionario forman parte de la muestra final un $82 \%$. Los restantes no contestaron al estudio o si lo hicieron lo rellenaron incorrectamente. En este sentido, el proceso de recogida de información ha sido por muestra no probabilística, ya que han seleccionado una muestra que representa a la población total periodística en prensa escrita en Almería. Se trata de un cuestionario autoadministrado enviado por mail. El cuestionario lo enviaron hasta en cuatro ocasiones durante los meses de junio, octubre y diciembre de 2010 y febrero de 2011.
} 
La autora enlaza las trayectorias de estos profesionales (algunos incluso con carreras extensas en el marco de los medios de comunicación tradicionales) con la crisis económica española, generadora de desempleo, que los lleva al desempleo y a buscar nuevas salidas, utilizando las TICS y creando micromedios locales. En este marco, muchos profesionales de la comunicación se convirtieron en emprendedores, creando su propio empleo, su propio micromedio.

En cuanto a los estudios latinoamericanos, las conclusiones arribadas son similares. Lagos y Cabalín (2009), realizaron 171 encuestas a editores y reporteros de los medios de comunicación de cobertura nacional con domicilio en la región metropolitana de Chile, con el objeto de identificar y describir las condiciones materiales, laborales y profesionales y la libertad de expresión de los comunicadores de dicha área geográfica. En su investigación, establecen una diferencia entre los periodistas y/o editores que recién comienzan a trabajar de aquellos que ya llevan un tiempo en el mercado laboral, afirmando que los primeros poseen condiciones contractuales más precarias que quienes llevan un tiempo en el mercado laboral, que poseen mayor tendencia a la estabilidad y formalidad laboral.

Estos autores establecen una diferencia en las condiciones laborales entre los medios tradicionales, como la prensa escrita, en la que los comunicadores de los medios poseen mejores condiciones laborales, en contraposición con las más precarios, que se dan en Internet y radio, en los que predominan los periodistas jóvenes. Por otro lado, la televisión es el medio de comunicación en donde se dan los mayores contrastes en cuanto a los salarios que pueden ser muy altos o muy bajos.

Por último, el estudio de Blanco (2016), analiza las trayectorias laborales de 74 entrevistados de las 3 cohortes de graduados de comunicación de la ECl, de Universidad Nacional de Córdoba, y concluye que tienden hacia la precarización laboral. En los 10 primeros tramos de empleo, el grupo más numeroso tiene trabajos eventuales (no registrados) y el segundo, contratos temporales sin aportes. En relación con los trabajadores en blanco y con estabilidad laboral, sólo son segundo grupo modal en los tramos 4, 7, 8 y 9.

A modo de síntesis, los estudios relevados dan cuenta de la situación de precariedad y desregulación legal en que la están inmersos los comunicadores sociales y periodistas, como sub-conjunto de los trabajadores. En el siguiente apartado se profundizará en el análisis de dichas problemáticas, desde el enfoque del derecho del trabajo.

\section{Abordaje de la problemática desde el enfoque del derecho del trabajo.}

La compilación y análisis del corpus bibliográfico abren el abanico para profundizar, en primer lugar, sobre el concepto de precariedad. En segundo lugar, sobre las modalidades y formas de contratación y relaciones laborales, desde el derecho individual: modalidad de contratación (contrato estable vs. temporal o por tiempo determinado), cantidad de horas trabajadas (jornada completa vs. jornada reducida), relación laboral (relación de dependencia vs. independiente o autónomo como contratación de servicios profesionales - periodísticos - telecomunicaciones - prensa - audiovisuales); y desde el derecho colectivo: sindicalización, convenios colectivos de trabajo, estatutos, estrategias de sociabilización; y desde la seguridad social y previsión (obra social, enfermedad, fallecimiento, desempleo o jubilación). 


\section{Definición por la OIT del trabajo precario.}

Desde los años 80 la precariedad laboral ha sido tema de interés para los países de la OCDE ${ }^{4}$. La OIT, a mediados de la década de los 90 y como consecuencia del aumento de la informalidad y el fenómeno de la globalización, comienza a analizar las relaciones laborales que luego serán catalogadas como precarias. En estos años analiza la mano de obra contratada, y bajo esta categoría a los contratos temporales, a aquellos con relaciones triangulares y a los autónomos; y sus relaciones encubiertas o ambiguas en las que no está claro quién es un asalariado y quien es un empleador.

La OIT realiza esta caracterización para diferenciarlos de las relaciones laborales que presentan un empleador conocido y que se encuentran bajo una relación de trabajo regulada y protegida por las normas nacionales e internacionales de trabajo. Sin embargo, si bien se visibilizó en el debate esta situación, culminó la negociación y no se llegó a un convenio o recomendación que los incluya (OIT, 2003; OIT, 2012).

Dado a que el trabajo precario es un asunto multifacético y complejo, por más que su término se emplee comúnmente su conceptualización resulta por demás compleja, y no existe una definición oficial con respecto a qué constituye un empleo precario. Muchos autores y actores sociales coinciden en que debe ser un término flexible que se adapte a las nuevas realidades que van surgiendo; muchos otros comparten la necesidad de definir lo que se entiende por empleo precario para permitir su regulación y la aplicación de políticas con la dirección a un sector predefinido, como así también para posibilitar la comparabilidad a los fines estadísticos.

En el simposio de ACTRAV, y en base a sus características se sostuvo que el trabajo precario es: "Un medio utilizado por los empleadores para trasladar los riesgos y las responsabilidades a los trabajadores. Es el trabajo que se realiza en la economía formal e informal y que se caracteriza por niveles variables y grados de particularidades objetivas (situación legal) y subjetivas (sensación) de incertidumbre e inseguridad. Si bien un trabajo precario puede tener diversas facetas, se lo suele definir por la incertidumbre que acarrea en cuanto a la duración del empleo, la presencia de varios posibles empleadores, una relación de trabajo encubierta o ambigua, la imposibilidad de gozar de la protección social y los beneficios que por lo general se asocian con el empleo, un salario bajo y obstáculos considerables tanto legales como prácticos para afiliarse a un sindicato y negociar colectivamente" (OIT, 2012, 32).

\section{Diferentes formas de condiciones y contrataciones laborales precarias que se vienen propagando.}

Una discusión clave en relación al futuro del trabajo tiene que ver con los cambios en la forma y relaciones de trabajo. Pasamos de un modelo de producción con una forma típica o clásica de empleo, como prevaleciente, en la cual los trabajadores empleados formalmente en relación de dependencia mantenían una subordinación económica, técnica y jurídica con su empleador y que se realiza a tiempo completo, a formas denominadas atípicas, no estandarizadas, que contemplan numerosas modalidades de trabajo modernas a las que se están adaptando cada vez mayor cantidad de trabajadores.

La expansión del empleo atípico no es un fenómeno nuevo, ni afecta por igual a todos los

4 Organización para la Cooperación y el Desarrollo Económicos es un organismo de cooperación internacional, compuesto por 38 estados, cuyo objetivo es coordinar sus políticas económicas y sociales. 
sectores de actividad, países ni regiones. Tampoco hay suficiente evidencia que permita suponer que en el futuro el empleo típico o estándar desaparecerá o que será reemplazado por el trabajo independiente. Lo cierto es que a esta preocupación se superpone, o se agrega, a un mercado de trabajo segmentado y con un alto porcentaje de precarización e informalidad (Novick, 2018).

La OIT distingue dos categorías de arreglos contractuales que se caracterizan por cuatro condiciones laborales precarias. i) Como arreglos contractuales enumera a la limitada duración del contrato (plazo fijo, corto plazo, temporal, estacional, trabajo a jornal y empleo ocasional); y la naturaleza de la relación de trabajo (relaciones de trabajo triangulares y encubiertas, falso autónomo, contratos de agencias o subcontratistas). ii) Y como condiciones precarias: salario bajo, escasa protección frente al despido, falta de acceso a la protección social y a los beneficios que se suelen asociar con un empleo estándar de tiempo completo, posibilidad limitada o nula de los trabajadores de ejercer sus derechos en su trabajo (OIT, 2012, 34).

\section{a) Modalidad de contratación (contrato estable vs. temporal o por tiempo deter- minado)}

“El empleo directo y permanente está en vías de extinción. Lo reemplazan progresivamente los contratos 'temporales', que de hecho pueden prolongarse durante décadas o por toda una vida." (OIT, 2012, 15). Los trabajadores con contratos temporales de diversa duración, se pueden beneficiar con el trabajo a corto plazo, pero tienen la incertidumbre de si el contrato se prolongará. Suelen ofrecer salarios más bajos y con desiguales beneficios a los contratos estables. Esta modalidad de contratación produce que el trabajador se encuentre en una situación de incertidumbre sobre su futuro y en cuanto a su protección social (OIT, 2012, 15; Novick, 2018).

\section{b) Cantidad de horas (jornada completa vs. jornada reducida)}

Tareas con una jornada inferior a las horas de trabajo de un trabajador full time, o empleo por tiempo parcial marginal o trabajo "por llamada" incluido contrato "0 hora" (Novick, 2018) También el trabajo en guardia o en espera.

El Convenio sobre el trabajo a tiempo parcial de la OIT N 175 (1994), no ratificado por la Argentina, establece que se deben adoptar medidas para asegurar que los trabajadores a tiempo parcial reciban la misma protección de que gozan los trabajadores a tiempo completo en situación comparable. Y si bien, es una forma de empleo atípico, nuestro país y varios países regulan la cantidad de horas máximas ${ }^{5}$. No obstante, lo que sucede es que, a pesar de su regulación, en el último tiempo han variado sus formas, reduciendo al máximo sus horas, con horarios impredecibles y trabajo "por llamada", sin obligación

\footnotetext{
5 Art. 92 ter LCT:1. El contrato de trabajo a tiempo parcial es aquel en virtud del cual el trabajador se obliga a prestar servicios durante un determinado número de horas al día o a la semana, inferiores a las dos terceras (2/3) partes de la jornada habitual de la actividad. En este caso la remuneración no podrá ser inferior a la proporcional, que le corresponda a un trabajador a tiempo completo, establecida por ley o convenio colectivo, de la misma categoría o puesto de trabajo. Si la jornada pactada supera esa proporción, el empleador deberá abonar la remuneración correspondiente a un trabajador de jornada completa. 2. Los trabajadores contratados a tiempo parcial no podrán realizar horas suplementarias o extraordinarias, salvo el caso del artículo 89 de la presente ley. La violación del límite de jornada establecido para el contrato a tiempo parcial, generará la obligación del empleador de abonar el salario correspondiente a la jornada completa para el mes en que se hubiere efectivizado la misma, ello sin perjuicio de otras consecuencias que se deriven de este incumplimiento. 3 . Las cotizaciones a la seguridad social y las demás que se recaudan con ésta, se efectuarán en proporción a la remuneración del trabajador y serán unificadas en caso de pluriempleo. En este último supuesto, el trabajador deberá elegir entre las obras sociales a las que aporte, a aquella a la cual pertenecerá. 4. Las prestaciones de la seguridad social se determinarán reglamentariamente teniendo en cuenta el tiempo trabajado, los aportes y las contribuciones efectuadas. Los aportes y contribuciones para la obra social será la que corresponda a un trabajador, de tiempo completo de la categoría en que se desempeña el trabajador. 5. Los convenios colectivos de trabajo determinarán el porcentaje máximo de trabajadores a tiempo parcial que en cada establecimiento se desempeñarán bajo esta modalidad contractual. Asimismo, podrán establecer la prioridad de los mismos para ocupar las vacantes a tiempo completo que se produjeren en la empresa.
} 
del empleador de garantizarle un número exacto de horas, lo que lo transforma en imprevisible.

c) Relación laboral (relación de dependencia vs. tercerización, independiente o autónomo como contratación de servicios profesionales - periodísticos - telecomunicaciones - prensa - audiovisuales).

El empleo denominado típico, directo o permanente, en el que se establece una relación binaria empleado-empleador, es sustituido por los contratos externos, por agencias o tercerizaciones; o bien por contratación de servicios u obras de autoempleados.

Como vimos con anterioridad, existen en la relación laboral, dos problemas que afectan a cada uno de los polos de la relación binaria. El primero es la falta de claridad en lo que respecta a la identidad del empleador, por ejemplo, los trabajadores que son contratados por una agencia o un subcontratista, pero desempeñan sus tareas para una empresa usuaria independiente. Estos trabajadores, se encuentran en una situación precaria cuando no está claro cuál de las dos partes debería responsabilizarse de los derechos y beneficios de los trabajadores.

El segundo, es la falta de claridad en la situación del empleado, ya que, si bien se vincula mediante una relación de contratación de servicios, en realidad lo que sucede es que se mantiene una relación de subordinación equivalente a la relación de dependencia. Estos trabajadores, se encuentran en una situación precaria porque no gozan de la protección y derechos laborales, y solo puede invocar la responsabilidad civil.

Estos marcos legislativos débiles y mecanismos de aplicación impotentes generan una situación en la que los trabajadores sometidos a una relación de trabajo encubierta o triangular casi no tienen forma de hacer valer sus derechos (OIT, 2012, 32-33).

d) Derecho colectivo: sindicalización, convenios colectivos de trabajo, estatutos, estrategias de sociabilización

La experiencia demuestra que con respecto a la acción gremial, sindical o colectiva es difícil que las disposiciones legales se materialicen o cumplan en la práctica. A través de la representación colectiva los trabajadores pueden negociar sus relaciones y condiciones de trabajo, con sus respectivas particularidades (OIT, 2012, 4), pero lo que sucede con el trabajo precario es que es difícil hacer valer los derechos sindicales ya que son escasos o incluso inexistentes.

En el simposio de la OIT ACTRAV se determinó de que: "Es preciso devolverle a los Convenios 87 y 98 su verdadero significado, que se viene vaciando de contenido a través de la propagación de las relaciones de trabajo precario. El alcance real de estos derechos se expresa en la árida frase unidad de negociación. La función de los convenios colectivos es la de rectificar una desigual relación de negociación. Por ende, se deduce que toda limitación, toda fragmentación de la unidad de negociación que aleja a los trabajadores de una relación de negociación con el verdadero empleador constituye una violación de derechos básicos" (OIT, 2012, 16).

e) Seguridad y previsión social (obra social, enfermedad, fallecimiento, desempleo 


\section{o jubilación)}

Los trabajos precarios también plantean problemas en los sistemas de seguridad social y previsional que se sustentan en una definición estricta de la relación de empleos típicos o clásicos basados en sistemas contributivos.

Los trabajadores precarios que no poseen registración, técnicamente no constan de un alta ante la AFIP, y por ende no realiza aportes ni contribuciones, al sistema previsional a los fines del acceso a la jubilación ni a las obras sociales que les permita contar con un seguro de salud.

Tampoco le es posible gozar de las asignaciones familiares contributivas, como son, la asignación por nacimiento, matrimonio, enfermedad, escolaridad, etc., y en el caso del despido tampoco podrá ser beneficiario del seguro de desempleo. Mucho menos, ser beneficiario de una ART que lo cubra frente a las contingencias laborales.

Por su parte, los trabajadores registrados, temporales, pueden tener problemas para constatar la existencia de una relación laboral para recibir esos derechos. Y los trabajadores autoempleados están habitualmente cubiertos solo por los riesgos más básicos, siendo ellos los responsables de los aportes y contribuciones.

\section{Marco legal que contempla a los comunicadores sociales y periodistas}

Como dijimos, las nuevas de formas de la organización del trabajo que afectan a los trabajadores en general y a los comunicadores y periodistas en particular, pueden reconfigurar las relaciones laborales: cada vez es más común que los trabajadores participen en una forma de empleo atípica (empleo temporal, empleo triangular, relaciones ambiguas y el trabajo a tiempo parcial). Esto puede resultar en un desafío para la aplicación de marcos normativos, ya sea en materia de protección o representación (OIT, 2016), produciéndose en la actualidad enormes vacíos legislativos o falta de regulación legal. A continuación, analizaremos puntualmente el marco normativo de los comunicadores sociales y periodistas.

\section{a) Normativa Internacional}

Con respecto a la actividad particular que ejercen los comunicadores y periodistas existen normas internacionales que los amparan en la particularidad del ejercicio de su profesión, sobre todo con respecto a su libertad de expresión, secreto profesional, rectificación, etc. Por ejemplo, la Declaración Universal de los Derechos Humanos que en su artículo 19 reivindica "el derecho de las personas a la libertad de opinión y expresión"; el Pacto Internacional de Derechos Civiles y Políticos, que protege el derecho para expresar opiniones e ideas también en su artículo 19; la Convención Americana sobre Derechos Humanos - Pacto Internacional de San José de Costa Rica articulo 13 y ss. que resguarda el derecho a la libertad de pensamiento y de expresión sin previa censura, con respeto a los derechos y la reputación de los demás, el derecho a rectificación o respuesta para personas afectadas por informaciones inexactas o agravantes.

Por otro lado, la Declaración de Principios sobre Libertad de Expresión adoptada por la Comisión Interamericana de Derechos Humanos (CIDH) de la OEA, que instruye a los Estados a garantizar el acceso a la información; que la censura previa debe estar prohibida por ley, que todo comunicador tiene derecho a la reserva de sus fuentes y que las leyes 
de privacidad no deben restringir la investigación. Y el Protocolo Adicional a los Convenios de Ginebra que incluye medidas de protección a periodistas.

Asimismo, como un subgénero de trabajadores se encuentran protegidos por las normas internacionales al igual que todos los otros trabajadores, como los Convenios, recomendaciones y resoluciones de la OIT, la Declaración Universal de Derechos Humanos, la Convención Americana de Derechos Humanos, etc.

\section{b) Normativa nacional}

En primer término, para poder analizar la regulación legal de los profesionales del área debemos decir que el ejercicio de su actividad se encuentra amparado por normas supremas y constitucionales.

De manera expresa, el artículo 14 de la Constitución Nacional (CN) protege el derecho de trabajar y la libertad de prensa6; el artículo 32 de la CN que se refiere a la libertad de imprenta ${ }^{7}$, artículo 42 de la CN que hace referencia a contrario sensu del deber de la prensa de brindar una información adecuada y veraz ${ }^{8}$, artículo 43 CN sobre el Habeas data y el secreto de las fuentes de información periodística ${ }^{9}$, artículo $68^{10}$ y $83^{11}$ de la CN con respecto a la inmunidad de los legisladores y publicación por la prensa respectivamente. De forma indirecta mediante los art.1 que establece la forma de gobierno, artículo 19 sobre la privacidad, artículo 23 que trata sobre el estado de sitio, artículo 28 que regula el principio de razonabilidad y artículo 33 que establece la forma republicana de gobierno.

Con respecto a normas de menor jerarquía es necesario diferenciar entre el comunicador social y el periodista. El primero no posee regulación legal alguna, lo que implica que no hay leyes que definan y contemplen las incumbencias de su actividad y no poseen colegiación ni matriculación como tales. Por su parte, el periodista dispone de un aparato legal que regula la actividad y por sobre todo su derecho de información y libertad de prensa, con posibilidad de matriculación ${ }^{12}$, tanto al que trabaja en relación de dependencia como al autónomo.

\footnotetext{
6 Derechos individuales, Art. 14:"Todos los habitantes de la Nación gozan de los siguientes derechos conforme a las leyes que reglamenten su ejercicio; a saber: De trabajar y ejercer toda industria lícita; de navegar y comerciar; de peticionar a las autoridades; de entrar, permanecer, transitar y salir del territorio argentino; de publicar sus ideas por la prensa sin censura previa; de usar y disponer de su propiedad; de asociarse con fines útiles; de profesar libremente su culto; de enseñar y aprender".

7 Libertad de Imprenta, Art. 32: "El Congreso federal no dictará leyes que restrinjan la libertad de imprenta o establezcan sobre ella la jurisdicción federal"

8 Derecho del consumidor, Art. 42: "Los consumidores y usuarios de bienes y servicios tienen derecho, en la relación de consumo, a la protección de su salud, seguridad e intereses económicos; a una información adecuada y veraz; a la libertad de elección, y a condiciones de trato equitativo y digno".

9 Habeas Data, Art. 43:"Toda persona podrá interponer esta acción para tomar conocimiento de los datos a ella referidos y de su finalidad, que consten en registros o bancos de datos públicos, o los privados destinados a proveer informes, y en caso de falsedad o discriminación, para exigir la supresión, rectificación, confidencialidad o actualización de aquellos. No podrá afectarse el secreto de las fuentes de información periodística".

10 Inmunidad de los legisladores, Art. 68: "Ninguno de los miembros del Congreso puede ser acusado, interrogado judicialmente, ni molestado por las opiniones o discursos que emita desempeñando su mandato de legislador".

11 Publicación por la prensa, Art. 83:"Desechado en todo o en parte un proyecto por el Poder Ejecutivo, vuelve con sus objeciones a la Cámara de su origen; ésta lo discute de nuevo, y si lo confirma por mayoría de dos tercios de votos, pasa otra vez a la Cámara de revisión. Si ambas Cámaras lo sancionan por igual mayoría, el proyecto es ley y pasa al Poder Ejecutivo para su promulgación. Las votaciones de ambas Cámaras serán en este caso nominales, por sí o por no; y tanto los nombres y fundamentos de los sufragantes, como las objeciones del Poder Ejecutivo, se publicarán inmediatamente por la prensa. Si las Cámaras difieren sobre las objeciones, el proyecto no podrá repetirse en las sesiones de aquel año".

12 Profundizando en la matrícula nacional de periodista que se justificará con el carné profesional que expedirá la autoridad administrativa del trabajo, que debe renovarse cada dos años y que si bien el estatuto dice que será de uso obligatorio, lo cierto es que en la actualidad ha perdido relevancia porque sirve para acreditar la identidad y trayectoria laboral pero se han derogado los beneficios y descuentos por lo que los periodistas prefieren acreditarse con la credenciales de los lugares para los que trabajan, antes de tramitarla o renovarla (César Arese, 2004).

En otro orden de ideas, César Arese en su artículo citando un fallo y abalado por numerosa doctrina y jurisprudencia nacional y precedentes internacionales dice que "admitir la condición de la matrícula y el carnet profesional para considerar que se trata de un periodista profesional, equivaldría a aceptar una licencia previa anticonstitucional y lesiva de la libertad de prensa" (TSJ de Córdoba, 24-6-71, D. T. XLI-935 en César Arese, 2004).
} 
Para poder analizar la regulación legal del comunicador o periodista no se tiene en cuenta el título profesional ${ }^{13}$ sino la actividad que realiza. Es así que aquellos profesionales que trabajen para las empresas de medios de comunicación en relación de dependencia se encuentran protegidos por la Ley de Contrato de Trabajo, ley 20.744 (en adelante LCT), y por el Estatuto del Periodista Profesional, ley $12.908^{14}$ (en adelante EPP), que establecen sus derechos y obligaciones, les da estabilidad y seguridad jurídica. Mientras que los profesionales que trabajan en áreas de comunicación institucional y/o comunitaria, organismos paraestatales y dependencias públicas que no realizan tareas periodísticas necesariamente, quedan desprotegidos del EPP, amparándose solo en la LCT, norma general que no contempla las especificidades de la profesión.

El EPP ostenta varias particularidades: “a) Es uno de los estatutos más completos y complejos del Derecho Laboral argentino ya que abarca aspectos de la actividad de prensa, matriculación profesional, condiciones de trabajo (período de prueba, jornada, vacaciones, suspensiones, etc.), extinción del nexo laboral y derecho colectivo; b) fue concebido y puesto en vigencia en sus aspectos esenciales hace sesenta años, por lo que desde su sanción han pasado varias olas tecnológicas transformando abismalmente el mundo de las comunicaciones (...) c) las relaciones entre los empleadores y los trabajadores de prensa, en tanto se desenvuelven dentro mismo de lo que se llama cuarto poder, no pueden considerarse únicamente desde el punto de vista estrictamente bilateral y laboral; hay fuertes componentes constitucionales cuando se trata de la libertad de prensa individual o colectiva, de protección del secreto y la fuente periodísticos; d) el EPP rige una profesión de alta exposición e influencia social" (César Árese, 2004, s.n).

Por otro lado, también existen comunicadores o periodistas que se definen como independientes y ofrecen sus servicios profesionales estableciendo una relación de igual a igual con quien los contrata. Entre ellos no existen una relación de dependencia y subordinación sino un contrato de locación de servicios (audiovisuales, telecomunicaciones, periodísticos, comunicación, etc.) que en principio se mantiene al margen de la regulación de la LCT y del EPP para regirse por el derecho civil. Decimos en principio, porque muchas veces se encubre una verdadera relación de dependencia bajo la contratación de servicios profesionales para vulnerar los derechos y la protección al trabajador dada por la LCT constituyendo un verdadero fraude laboral.

\section{Conclusión}

Los cambios en el mundo del trabajo y las nuevas formas de la organización del mismo, afectan a los trabajadores en general y a los comunicadores y periodistas en particular, y reconfiguran las relaciones laborales, Como vimos, cada vez resulta más común que los trabajadores participen de una forma de empleo atípica, hoy catalogada en su mayoría como precaria e informal.

\footnotetext{
13 Para el ejercicio del periodismo y algunas actividades de comunicación siquiera no es requisito poseer un título profesional.

14 Art. $2^{\circ}$ - Se consideran periodistas profesionales, a los fines de la presente ley, las personas que realicen en forma regular, mediante retribución pecuniaria, las tareas que les son propias en publicaciones diarias, o periódicas y agencias noticiosas. Tales el director, codirector, subdirector, jefe de redacción, secretario general, secretario de redacción, prosecretario de redacción, jefe de noticias, editorialista, corresponsal, redactor, cronista, reportero, dibujante, traductor, corrector de pruebas, reportero gráfico, archivero y colaborador permanente. Se incluyen las empresas radiotelefónicas, cinematográficas o de televisión que propalen, exhiban o televisen informativos o noticias de carácter periodístico, y únicamente con respecto al personal ocupado en estas tareas. Se entiende por colaborador permanente aquel que trabaja a destajo en diarios, periódicos, revistas, semanarios, anuarios y agencias noticiosas, por medio de artículos o notas, con firma o sin ella, retribuidos pecuniariamente por unidad o al centímetro, cuando alcance un mínimo de veinticuatro colaboraciones anuales.

Quedan excluidos de esta ley los agentes o corredores de publicidad y los colaboradores accidentales o extraños a la profesión. No se consideran periodistas profesionales los que intervengan en la redacción de diarios, periódicos o revistas con fines de propaganda ideológica, política o gremial, sin percibir sueldos
} 
Los trabajadores precarios si bien forman parte de los "ocupados"15 en las estadísticas de medición de los organismos oficiales como las de INDEC ${ }^{16}$, OIT, CEPAL ${ }^{17}$, etc., estos trabajadores presentan cero o ineficiente protección legal, cobertura social y previsional.

Entonces, no basta solo con decir que están ocupados, sino que dicha ocupación debería ser decente. La OIT define el trabajo decente como aquel "que resume las aspiraciones de la gente durante su vida laboral. Significa contar con oportunidades de un trabajo que sea productivo y que produzca un ingreso digno, seguridad en el lugar de trabajo y protección social para las familias, mejores perspectivas de desarrollo personal e integración a la sociedad, libertad para que la gente exprese sus opiniones, organización y participación en las decisiones que afectan sus vidas, e igualdad de oportunidad y trato para todas las mujeres y hombres".

En este contexto, resulta un desafío reinventar la normativa y las leyes del trabajo para regular las nuevas relaciones laborales. ya que existe un quiebre entre el trabajo y las protecciones. Como se ha analizado, esto es particularmente relevante para los comunicadores y periodistas, muchos de los cuales se mantienen relaciones de empleo flexibles, que redundan en condiciones de precariedad.

Como conclusión, la crisis del trabajo que atravesamos es también una crisis jurídica, porque se pone en duda la capacidad del derecho para regular las nuevas formas y modalidades contractuales. Existe un quiebre, entre quienes piensan que un derecho del trabajo demasiado rígido es un obstáculo para el desarrollo económico y del trabajo; y entre quienes consideran que es necesario la regulación legal de la nueva realidad porque ha sido el derecho el que históricamente les ha otorgado dignidad a los trabajadores. Comparto esta última posición, y considero necesario visibilizar la realidad, formular nuevas normas laborales que la contemple y propender a una rearticulación de políticas macroeconómicas con políticas sociales y laborales, además de programas específicos para los grupos más vulnerables.

\footnotetext{
15 Personas ocupadas: son aquellas que tienen más de cierta edad especificada y que durante el breve periodo de referencia de la encuesta (que puede ser una semana, mes o trimestre) estuvieron ocupadas al menos una hora: (1) con empleo asalariado -es decir, trabajando durante el periodo de referencia por un sueldo o salario-, u ocupadas pero sin trabajar debido a una ausencia temporal en el periodo de referencia durante la cual mantuvieron un vínculo formal con su puesto de trabajo; o (2) con empleo independiente, trabajando para obtener beneficios o ganancia familiar (incluye a los trabajadores familiares auxiliares), o sin trabajar por una ausencia temporal durante el periodo de referencia. En CEPAL (2019). Anuario Estadístico de América Latina y el Caribe. Naciones Unidas. Santiago.

Recuperado de: https://www.cepal.org/es/publicaciones/ae

16 Instituto Nacional de Estadística y Censos - INDEC Argentina.

17 La Comisión Económica para América Latina y el Caribe es el organismo dependiente de la Organización de las Naciones Unidas responsable de promover el desarrollo económico y social de la región.
} 


\section{Referencias Bibliográficas}

- Arese, C. (2004). El estatuto del periodismo profesional: desde el dictáfono a Internet, en Revista de Derecho Laboral. II Doctrina- Jurisprudencia- Actualidad. Rubinzal-Culzoni Editores.

- Baranchuk, M. (2016). ¿Quo Vadis trabajador de la comunicación y la cultura? Sociales en debate $\mathrm{N}^{\circ} 10$. Facultad de Ciencias Sociales. Universidad de Buenos Aires. Recuperado de: http://www.sociales.uba.ar/wp-content/blogs.dir/219/files/2016/08/Art7BARANCHUK.pdf

- Blanco, C. (2016). Trayectorias de formación y de trabajo de graduados de comunicación de la UNC en un contexto heterogéneo. Un estudio longitudinal. (Tesis de Doctorado). Doctorado en Estudios Sociales de América Latina, Centro de Estudios Avanzados (CEA), Universidad Nacional de Córdoba.

- Castel, R. (2012). El ascenso de las incertidumbres. Trabajo, protecciones, estatuto del individuo. Buenos Aires: Fondo de Cultura Económica.

- Chinea Rodríguez, M. S. (2013). TIC y transformaciones de las biografías laborales: los comunicadores en Canarias. Santa Cruz de Tenerife. Universidad Nacional de Educación a Distancia - UNED. Facultad de Educación. Trabajo Fin del Máster Universitario Comunicación y Educación en la Red: de la sociedad de la información a la sociedad del conocimiento. Especialidad: Subprograma de investigación en tecnologías digitales en la sociedad del conocimiento.

- Lagos, C. y Cabalín, C. (2009). Condiciones profesionales del ejercicio del periodismo y de la libertad de expresión en los principales medios de comunicación de la región Metropolitana. Santiago de Chile: ICEI.

- Martínez, S. (2019). Condiciones laborales de los periodistas. Incidencia en la práctica profesional. (Tesis de posgrado). Universidad Nacional de Quilmes, Bernal, Argentina. Disponible en RIDAA-UNQ Repositorio Institucional Digital de Acceso Abierto de la Universidad Nacional de Quilmes. Recuperado de: http://ridaa.unq.edu.ar/handle/20.500.11807/1745

- Novick, M. (2018), El mundo del trabajo: cambios y desafíos en materia de inclusión. Naciones Unidas - CEPAL. Serie Políticas Sociales 228 (LC/TS.2018/2). Recuperado de https://www.cepal.org/es/publicaciones/43221-mundo-trabajo-cambios-desafios-materia-inclusion

- OIT (2003). Memorándum: 91.a reunión de la Conferencia Internacional del Trabajo. Ginebra: OIT. 3 al 19 de junio. Recuperado de: https://www.ilo.org/public/spanish/standards/relm/ilc/ilc91/pdf/memo.pdf

- OIT (2012). Del trabajo precario al trabajo decente: documento final del simposio de los trabajadores sobre políticas y reglamentación para luchar contra el empleo precario. Oficina Internacional del Trabajo, Oficina de Actividades para los Trabajadores (ACTRAV). Ginebra: OIT. Recuperado de https://www.ilo.org/wcmsp5/groups/public/@ed_dialogue/@ actrav/documents/meetingdocument/wcms_179789.pdf

- Ufarte Ruiz, M. J. (2011). El periodista acosado: entre la precariedad laboral y el mobbing. Un estudio de caso: la precariedad de los periodistas almerienses en la prensa escrita (Tesis de doctorado). Universidad de Sevilla, Sevilla, España.

- Rodríguez E. y Sánchez R. (2004). Prólogo: Entre el capitalismo cognitivo y el Commonfare, En Capitalismo cognitivo, propiedad intelectual y creación colectiva. Madrid: traficantes de sueños, 13-28. 